\title{
Long-term Monitoring of Solar Activity by Amateur Astronomers
}

\author{
Klaus Reinsch \\ Wilhelm-Foerster Sternwartem Munsterdamm 90, D-1000, Berlin 41, Germany
}

\section{Introduction}

Professional solar astronomy concentrates on the study of the atmosphere and interior of the Sun. Little attention is given to "classical" programmes, mainly statistical investigations of solar activity. Although the main properties of phenomena associated with the solar cycle seem to be understood there are still enough details to be explained, making it worthwhile monitoring different indicators of solar activity, even if no immediate results are to be expected. Such routine observations are ideal work of amateur astronomers.

Members of West German local astronomical societies founded the journal Sonne in 1977 to combine their efforts on solar observations. The first issue was presented at a conference on amateur solar observation held in Berlin in April 1977. Sonne is compiled by an editorial staff of 23 amateurs from all over West Germany, and is distributed among nearly 500 readers in 20 countries. With the increasing number of foreign readers, the main articles in Sonne are provided with English abstracts.

Common efforts of 28 amateurs led to another remarkable product: in 1983 the "Handbook for Solar Observers" was completed after 5 years work. In 700 pages all topics of current amateur solar observation are discussed: white light, $\mathrm{H} \alpha$, radio, and solar eclipses, including all relevant techniques and references. An English translation is in preparation.

\section{Observing Programmes}

The staff of Sonne also coordinate different observing programmes:

Sunspot numbers; new sunspot indices; daily charts of the Sun; sunspot positions; white-light faculae; solar photography; $\mathrm{H} \alpha$ observations; light bridges; naked-eye sunspot observations; solar eclipses.

The sunspot network currently has more than 130 observers worldwide. Daily Wolf sunspot numbers are evaluated from about 17000 individual observation per year. The basis for the reduction of the sunspot numbers are the data obtained by a group of experienced and qualified observers who are selected yearly. Definitive sunspot numbers now cover nearly a complete cycle (see Fig. 1). Smoothing of the monthly means is done by an improved method using a polynomial weighting function over 17 months (instead of the traditional box-like weighting function). 


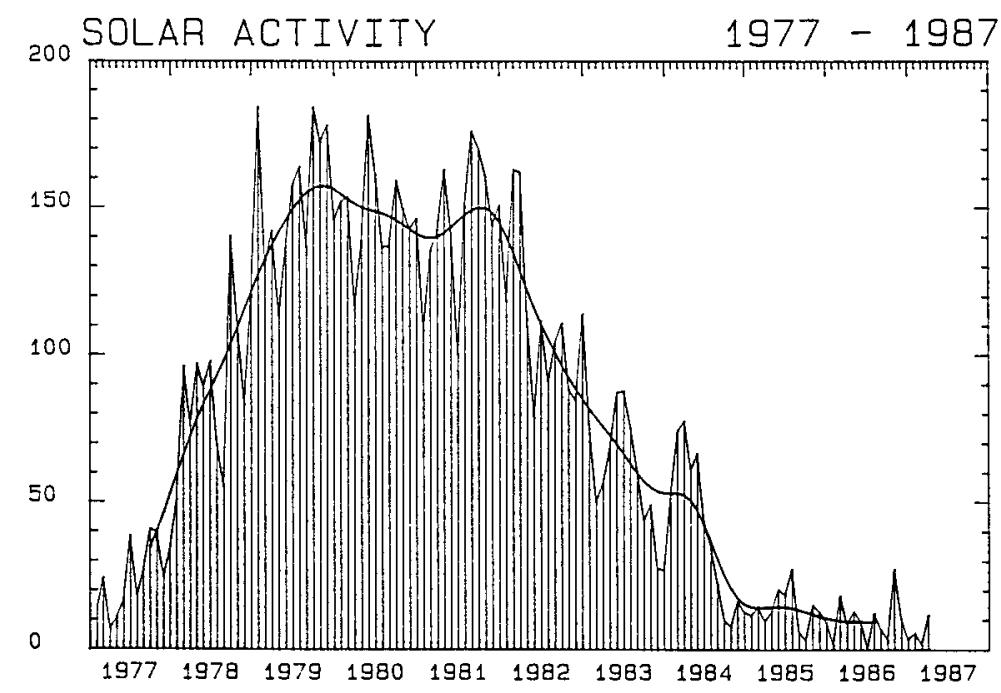

Fig. 1. Monthly and smoothed means of the definitive sunspot numbers evaluated by the Sonne network

The comparison between the SIDC and the Sonne sunspot numbers is shown in Fig. 2. Although there is a good match overall, the Sonne sunspot numbers tend to lie above the SIDC values at high activity and below them at low activity. But a similar relation is noted when comparing the SIDC data with the sunspot numbers obtained by the Zurich observatory, which was been the international standard until 1980 .

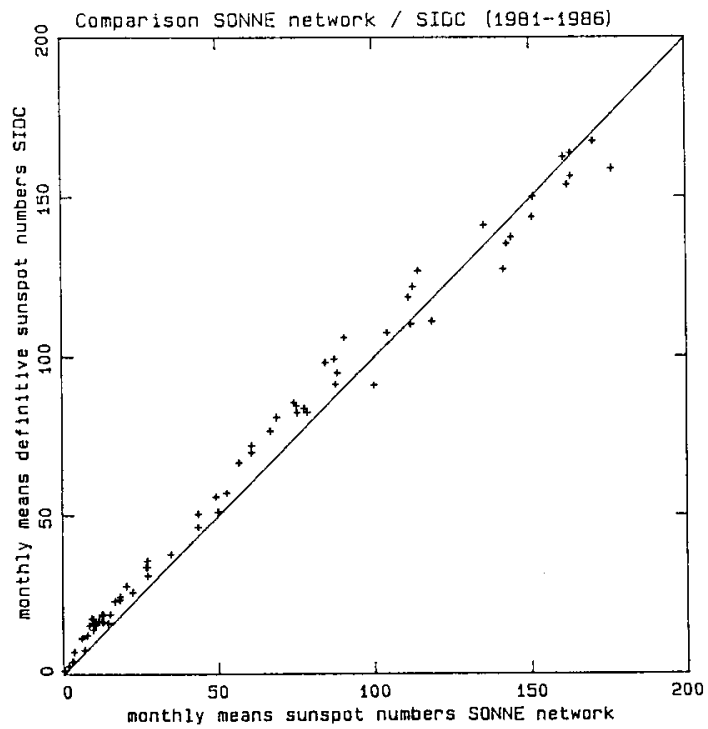

Fig. 2. Comparison between SIDC and Sonne sunspot numbers (1981-1986) 
It is well known that the sunspot number defined by Wolf is not the best measure of solar activity, because small and large sunspot groups are given the same weighting factor of 10 . A new sunspot number that uses different weighting factors according to the Waldmeier classification of a group, has been introduced by R. Beck. This gives an estimate of the sunspot area:

$$
\operatorname{Re}=\sigma_{i} G_{i} f_{i}
$$

with the weighting factors defined as quotients of mean sunspot area / mean sunspot number-

\begin{tabular}{lllllllllll}
\hline Waldmeier classification & A & B & C & D & E & F & G & H & J \\
weighting factor $G_{i}$ & 4 & 4 & 8 & 18 & 25 & 36 & 50 & 44 & 37 \\
\hline
\end{tabular}

has been tested by a group of observers over several years. The results are compared with measurements of the projected sunspot area.

New classification schemes were developed for white-light faculae, light bridges, and prominences, as a base for long-term statistical investigations. Facular activity is measured by the number of faculae groups visible. A distinction is made between faculae accompanied by spots, and faculae without spots. First results based on observations from 1979 to 1986 show that the modulation in the total number of faculae groups is caused by faculae that surround spots (Fig. 3).

Measurements of sunspot positions allow activity in northern and southern latitudes to be distinguished. A significant surplus of spots at southern latitudes was found from 1983 to 1985 (Fig. 4). Synoptic charts of the photosphere show the distri-

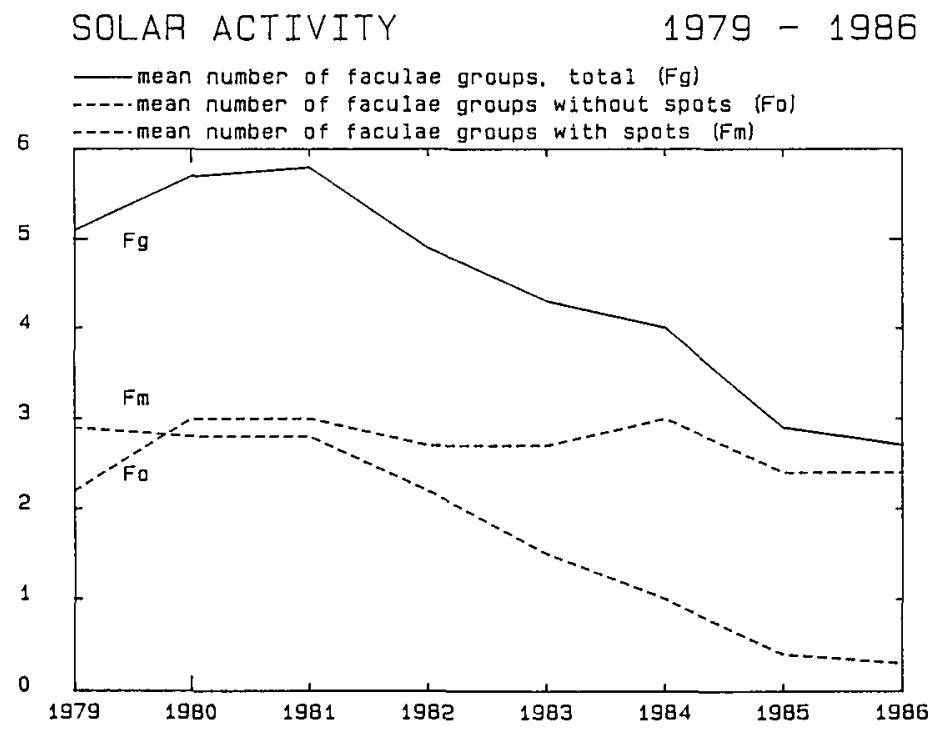

Fig. 3. Facular activity measured by the number of faculae groups visible (based on evaluations by V. Gericke) 


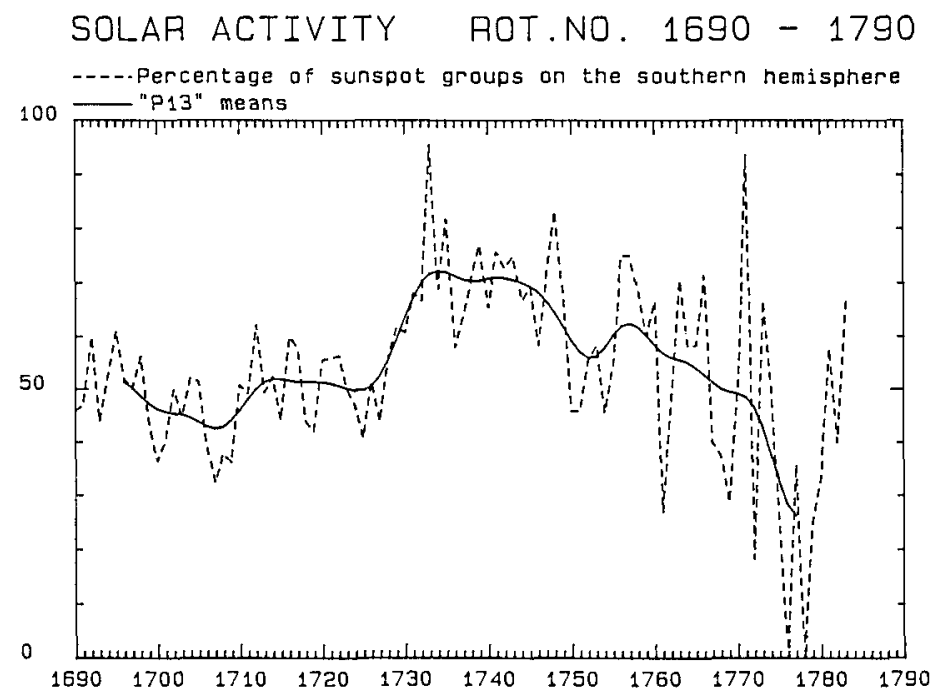

Fig. 4. Percentage of sunspot groups on the southern hemisphere (based on computations by E. Junker)

bution of spots in longitude and latitude. These charts are drawn for each Carrington rotation, and yearly as a summary diagram. The solar "butterfly diagram" published by professional authors until 1976 is continued by amateur work.

Photographic observations in white light, $\mathrm{H} \alpha$ and $\mathrm{Ca}$ II $(\mathrm{K})$ are used, e.g. to simultaneously study the development of active regions. For example, the large sunspot group in June 1983 was analysed in detail, confirming a relation between proper motions within a group and flare activity.

\section{Acknowledgements}

I wish to thank Günter Appelt, Dr. Rainer Beck, Volker Gericke, Sieglinde Hammerschmidt, Cord-Hinrich Jahn and Elmar Junker for contributing diagrams and results of their work to this paper. I also acknowledge the kind assistance of Stefan Haacke who provided the French abstract for the conference programme. 